\title{
Efficiency of Supplementary Contemporary Single-file Systems in Removing Filling Remnants from Oval-shaped Canals: An In Vitro Study
}

\author{
Neveen A Shaheen ${ }^{1}$, Nahla G Elhelbawy ${ }^{2}$, Dalia A Sherif ${ }^{3}$
}

\begin{abstract}
Aim: This study investigated the performance of supplementary three single-file systems for eliminating root filling residue from oval-shaped canals utilizing cone-beam computed tomography (CBCT).

Materials and methods: Thirty single-rooted human mandibular premolars with oval-shaped canals were chosen. After decoronation and working length determination, ProTaper Universal (PTUR) rotary files were used to prepare canals up to F3. Irrigation was fulfilled by $2.5 \%$ sodium hypochlorite $(\mathrm{NaOCl}), 17 \%$ ethylenediaminetetraacetic acid (EDTA), and lastly distilled water. By utilizing the lateral compaction technique, canals were filled by gutta-percha and Endosequence BC Sealer. The PTUR retreatment system (D1, D2, and D3) eliminated the primary filling material sequentially. Subsequently, specimens were categorized into three groups at random: group I: WaveOne Gold (WOG) primary file, group II:TruNatomy (TRN) medium file, and group III: XP-endo Finisher R (XPF-R). The volume of residual left from filling material was quantified utilizing CBCT imaging before and after the supplementary retreatment. Statistical analyses were done by one-way analysis of variance (ANOVA) and Tukey's test at a 5\% significance level.

Results: The volume of removed root filling after using XPF-R was significantly higher than using WOG in all sections and TRN in the coronal third, while nonsignificantly higher than using TRN in the apical and middle.

Conclusion: XPF-R as a supplementary technique revealed the highest percentage reduction of root filling material (73.13\%) in comparison with the other systems ( $37.39 \%$ WOG and $44.16 \%$ TRN) in oval-shaped canals.

Clinical significance: Neither one of the methods and tools used to remove filling material from oval-shaped root canals is perfectly efficient. The performance of supplementary techniques using contemporary single-file systems improves the cleaning and reshaping of root canal space that permits a proper disinfection to optimize the outcome of retreatment option.

Keywords: Filling remnants, Oval-shaped canal, TruNatomy, WaveOne Gold, XP-endo Finisher R.

The Journal of Contemporary Dental Practice (2021): 10.5005/jp-journals-10024-3198
\end{abstract}

\section{INTRODUCTION}

A common cause of posttreatment disease is persistent or secondary intraradicular infection. ${ }^{1}$ When the primary therapy fails, the clinician recommends nonsurgical root canal retreatment. Canal retreatment entails removing all old filling material to shape, disinfect, and fill the entire endodontic system thoroughly. ${ }^{2,3}$ Effective removal is a technical hurdle with significant clinical implications enhancing better cleaning and disinfection. ${ }^{4}$ Some of the options utilized to remove the previous filling from root canals are hand files, nickel-titanium (NiTi) rotary instruments, Gates Glidden, heated instruments, ultrasonic, laser, and solvent. Regardless of the instrument designs and alloys or techniques utilized during retreatment, residual debris remain in the canal walls and it is difficult to be removed totally. ${ }^{5-13}$ As a result, supplementary procedures have been devised that are mainly created to enhance the removal of existing root filling materials and improve root canal disinfection.

New single-file systems have been developed as a result of recent advancements in endodontic instrumentation in terms of design and metal treatment. WaveOne Gold (WOG), XP-endo Finisher R (XPF-R) instrument, and TruNatomy (TRN) instruments are some of the most current single-file systems in the market. Because of their enhanced mechanical characteristics, they could be appropriate for root canal retreatment. WOG is a single-file NiTi system with a reciprocating motion that features novel designs

\footnotetext{
1,3Endodontic Department, Faculty of Dentistry, Tanta University, Tanta, Egypt

${ }^{2}$ Dental Biomaterials Department, Faculty of Dentistry, Tanta University, Tanta, Egypt

Corresponding Author: Nahla G Elhelbawy, Dental Biomaterials Department, Faculty of Dentistry, Tanta University, Tanta, Egypt, Phone: +201006422121, e-mail: nahla_elhelbawy@dent.tanta.edu.eg

How to cite this article: Shaheen NA, Elhelbawy NG, Sherif DA. Efficiency of Supplementary Contemporary Single-file Systems in Removing Filling Remnants from Oval-shaped Canals: An In Vitro Study. J Contemp Dent Pract 2021;22(9):1055-1059.

Source of support: Nil

Conflict of interest: None
}

and metallurgy. It is made of thermally treated NiTi, called Gold $\mathrm{NiTi}$ alloy. This file system is featured by a parallelogram crosssection, greater flexibility than the original WaveOne instruments, variable tapers, and a semi-active tip. ${ }^{14,15}$ It has superior mechanical properties and is more efficient during the filling material removal from straight canals than the full-sequence rotary NiTi system. ${ }^{16-18}$

The XPF- $R$ is a single-file system with continuous rotation motion designed exclusively for retreatment. It is a variant of XPF that possesses a broader core diameter of 30 and a zero-taper, 
making it stiffer and more aggressive in displacing root filling materials that remain after conventional methods. Furthermore, it is made of MaxWire alloy, which is morphologically altered at body temperature, assuming a spoonlike shape at its active tip that expands and contracts to adapt to canal morphology with a reach of $6 \mathrm{~mm}$ in diameter or 100-fold larger than a standard instrument of the same size. The supplementary approach with the XPF-R improved the removal of existing filling material in some studies. $^{15,19-22}$

TRN instruments are a new kind of heat-treated NiTi instrument and have a unique recently invented design. TRN instruments come in three sizes: small (20/0.04), prime (26/0.04), and medium $(36 / 0.03)$. These files have a variable regressive taper and an off-center parallelogram square cross-sectional design. Different heat treatments have been applied to these files, enhancing their elasticity and resistance to cyclic fatigue. ${ }^{23-25}$ No data was available for using TRN as a supplementary approach for removing old root canal filling.

Consequently, the aim of this study was to assess the performance of three contemporary single-file systems (WOG, TRN, and XPF-R) when used as a supplementary approach to remove remaining root filling residues from oval-shaped canals after basic retreatment utilizing cone-beam computed tomography (CBCT) analysis. The following hypotheses were investigated: Supplementary approaches improve the removal of root filling materials after basic retreatment; however, there are no differences between the three single-file systems in volume of removed root filling materials.

\section{Materials and Methods}

The Research Ethics Committee of the Faculty of Dentistry, Tanta University, authorized the study plan. The study was conducted in clinics and research center of the Faculty of Dentistry, Tanta University. Thirty single-rooted human mandibular premolars with a single oval-shaped root canal recently extracted for orthodontic purposes were chosen. Teeth with multiple canals, open apices, root caries, cracks, internal resorption, external resorption, or calcification were discarded. Shortly after extraction, the teeth were washed with sterile saline and kept individually in a $10 \%$ buffered formalin solution. Immediately before use, they were thoroughly washed under running tap water. Digital radiographs were obtained from the mesiodistal and buccolingual projections to verify that the selected root canals were single, oval-shaped, and straight. The coronal parts of all teeth were eliminated with a water-cooled diamond disk (Komet; Brasseler, Lemgo, Germany), providing a $14 \pm 1$-mm-long root segment. The working length was determined using a 10-stainless steel file (Mani Incorporation, Tochigi, Japan). It was inserted into the canal until its tip was seen at the apical foramen and then subtracted $1 \mathrm{~mm}$.

ProTaper Universal (PTUR) rotary instruments (Dentsply Maillefer, Ballaigues, Switzerland) were utilized to prepare the root canals up to F3 (30/0.06). The canals were irrigated with a 30-gauge side-vented NaviTip (Ultradent, South Jordan, Utah, USA) with $20 \mathrm{~mL}$ of $2.5 \%$ sodium hypochlorite (Clorox Company, 10th of Ramadan, Egypt), followed by $3 \mathrm{~mL}$ of $17 \%$ ethylenediaminetetraacetic acid (EDTA) for 3 minutes to remove the smear layer. Subsequently, the canals were rinsed with $2 \mathrm{~mL}$ distilled water before they were dried with paper points (DiaDent Group International, Burnaby, B.C. Canada). The root canal was obturated with a size 30, 0.06 taper master guttapercha cone (Meta Biomed, Pennsylvania, USA) that was coated with
Endosequence BC Sealer (Brasseler USA, Savannah, Georgia, USA) and inserted inside the canal until reaching a full working length (WL). Cold lateral compaction was accomplished using a size 25 finger spreader and accessory gutta-percha cones (size 20, 0.02 taper).

A digital radiograph of both the buccolingual and mesiodistal projections was utilized to verify the efficiency of the root filling. Samples with unfulfilling root canal filling were dismissed and replaced with comparable features. A cotton pellet and a cavit were utilized to temporarily seal the access cavities (ESPE, Seefeld, Germany). Subsequently, the specimens were kept for 7 days at $37^{\circ} \mathrm{C}$ and $100 \%$ humidity to allow for a sealer set.

\section{Retreatment Techniques}

PTUR retreatment system (Dentsply Maillefer, Ballaigues, Switzerland) D1 (30/0.09), D2 (25/0.08), and D3 (20/0.07) files were utilized in a pecking motion to eliminate the whole root canal filling material, respectively, till the working length established with D3. The complete removal of the filling materials was verified by obtaining the working length, no longer filling materials removed by the instruments, and finally via radiograph. An X-Smart Plus motor (Dentsply, Tulsa, Oklahoma, USA) was used with all files at a constant speed of $500 \mathrm{rpm}$ and a torque of $3 \mathrm{~N} \mathrm{~cm}$. After performing basic retreatment procedure using PTUR, the specimens were scanned to calculate the volume of filling material using the following parameters, CBCT scan images in axial, coronal, and sagittal plans were captured using Cranex 3D device (Soredex, Helsinki, Finland) operating at $90 \mathrm{kV}$ and $8 \mathrm{~mA}$, and exposure parameters were kept constant before and after the supplementary approaches. ${ }^{26}$

\section{Supplementary Removal Technique}

Subsequently, specimens were divided into three groups $(n=10)$ based on the supplementary retreatment tools utilized:

Group I: WOG primary file (Dentsply, Maillefer, Switzerland) (tip size $25,0.07$ ) was operated in the X-Smart Plus motor using the preset reciprocation mode.

Group II:TRN medium (Dentsply, Sirona, Ballaigues, Switzerland) size 36 and $3 \%$ taper was operated at $500 \mathrm{rpm}$ with a torque setting of $1.5 \mathrm{~N} \mathrm{~cm}$.

Group III:XPF-Rinstrument (FKG Dentaire SA, La Chaux-de-Fonds, Switzerland) (tip size 30, 0.0 taper) was operated in the same motor at $800 \mathrm{rpm}$ and $1 \mathrm{~N} \mathrm{~cm}$ torque.

The plastic tube enclosing the instrument was cooled with ethyl chloride spray (Walter Ritter, Pharmaceutical, Germany) to maintain the files straight while measuring the WL. The file was put in the root canal without being rotated, and then, it was activated. It was pressed against the sidewalls of the canals in three cycles; each cycle is composed of in-and-out motions with an amplitude of 7-8 $\mathrm{mm}$, up to the WL. This technique was carried out in a cabinet with a heater and thermometer to maintain a temperature of $37^{\circ} \mathrm{C}$ to imitate body temperature and provide proper circumstances for phase change of XPF-R instruments.

\section{Statistical Analysis}

The difference between the original volume of the residual root filling material after retreatment (baseline) and the final root filling material volume following the use of the three supplemental approaches was used to calculate the volume of residual filling material $\left(\mathrm{mm}^{3}\right)$. The volume of root filling material reduction was also calculated as a percentage. The volume of residual filling material at both time points (following the basic and final 
supplementary protocols) was analyzed using one-way ANOVA followed by Tukey's test for pairwise comparisons. Differences between the groups were considered statistically significant when $p \leq 0.05$. All statistical analyses were performed using SPSS version 20 (SPSS, Chicago, Illinois, USA). The volume of residual filling material was analyzed using one-way ANOVA followed by Tukey's test for pairwise comparisons at both time points (after the basic and final supplemental protocols). When $p \leq 0.05$, differences between the groups were considered statistically significant. SPSS version 20 was used for all statistical analyses (SPSS, Chicago, Illinois, USA).

\section{Results}

The mean remaining filling material volume after basic retreatment using PTUR was comparable for all groups $(p=0.9702)$. A varying amount of residual filling material was observed along the entire length of the canal wall in all supplementary retreatment groups ( $p$ <0.0001). A summary of the mean volume of residual filling material is shown in Table 1. After using the supplementary retreatment technique, when comparing between the groups regardless of root canal sections, XPF-R removed significantly more root filling material $(p<0.0001)$. It recorded the least volume of residual filling material $(3.12 \pm 1.41)$, while WOG and TRN recorded $6.69 \pm 1.58$ and $5.91 \pm 1.62$, respectively, with no statistically significant difference between them. Table 1 shows that the apical third after XPF-R possesses the least mean volume of the residual filling material $(1.55 \pm 0.47)$ and the highest value recorded in the coronal third after using WOG $(8.17 \pm 0.89)$.

When comparing between root canal sections regardless of groups, there was a significant difference in the volume of remaining filling material in the root canal thirds by either PTUR ( $p<0.0001)$ or supplementary approaches $(p=0.0008)$. The least volume of remaining filling material was recorded for the apical third $(8.34 \pm 3.89,3.89 \pm 1.92)$ in comparison with the middle $(10.52 \pm 1.05,5.15 \pm 1.69)$ and coronal thirds $(14.59 \pm 1.46,6.69 \pm 1.98)$ after basic and supplementary approaches, respectively. The highest volume of the removed filling material was recorded for XPF-R $(8.15 \pm 1.98)$, while WOG and TRN recorded $4.31 \pm 2.14$ and $5.26 \pm 2.56$, respectively. Table 2 demonstrates that the mean volume of removed root filling after using XPF- $R$ was significantly higher than using WOG in all sections and TRN in the coronal third, while nonsignificantly higher than using TRN in the apical and middle.

XPF-R revealed the highest percentage of reduction of root filling material $(73.13 \%)$ in comparison with the other systems (37.39\% WOG and $44.16 \%$ TRN) with statistically significant difference ( $p<0.0001)$ when considering remaining filling material after basic PTUR retreatment technique as 100\%. Table 3 showed that the percentage reduction of root fillings after using XPF- $R$ was significantly higher than that of the other single-file systems in the apical and coronal third and WOG in the middle third. However, it was nonsignificantly higher than that of TRN in the middle third.

\section{Discussion}

Root canal retreatment attempts to eliminate previous filling material as it acts as a physical barrier to the activity of irrigation solutions and intracanal medicaments on the infected dentine of the root canal space. ${ }^{3}$ The oval-shaped canals possess anatomic and morphologic irregularities, such as fins, recesses, and isthmuses, which make them difficult and affect the capabilities of instruments or approaches to eliminate filling material because filling materials originally flowed into these irregularities were difficult to be reached by the instruments. ${ }^{27}$ It is a challenge either in the preparation of the canal or in the removal of the old filling; therefore, in this study mandibular premolars with oval-shaped canals were chosen.

Endosequence BC Sealer was chosen in the current study as a new trend toward the use of bondable obturating materials has gained popularity due to its biocompatibility and increased adherence to root dentin. Moreover, the removal of the filling materials from the root canal using this sealer is a challenge since it is hard upon setting. None of the existing retreatment procedures were successful in entirely removing the whole root canal filling material. In this investigation, retreatment was used to remove most root filling material using PTUR retreatment files (D1-D3), followed by supplementary tools to improve filling material removal. CBCT is a nondestructive 3D imaging technology that allows a longitudinal inspection of the same specimen through numerous experimental procedures at various times. In this research, it was utilized to assess the implication of using supplementary files in removing the remaining filling material from oval-shaped canals as in the previous research. ${ }^{15,18}$

Table 2: Mean and standard deviation of the volume of removed root filling (volume after basic retreatment technique-volume after supplementary retreatment techniques) in all groups along the entire root canal length

\begin{tabular}{lcccc}
\hline & \multicolumn{3}{c}{ Volume of removed root fillings } & \\
\cline { 2 - 4 } & Group I & Group II & Group III & p value \\
\hline Apical & $2.79 \pm 2.00^{\mathrm{A}, \mathrm{a}}$ & $3.61 \pm 2.89^{\mathrm{C}, \mathrm{a}, \mathrm{b}}$ & $6.94 \pm 1.64^{\mathrm{D}, \mathrm{b}}$ & 0.0297 \\
Middle & $3.86 \pm 1.53^{\mathrm{A}, \mathrm{B}, \mathrm{e}}$ & $5.00 \pm 2.28^{\mathrm{C}, \mathrm{e}, \mathrm{f}}$ & $7.21 \pm 0.46^{\mathrm{D}, \mathrm{f}}$ & 0.0193 \\
Coronal & $6.26 \pm 1.35^{\mathrm{B}, \mathrm{i}}$ & $7.16 \pm 1.18^{\mathrm{C}, \mathrm{i}}$ & $10.32 \pm 1.42^{\mathrm{E}, \mathrm{j}}$ & 0.0010 \\
$p$ value & 0.0176 & 0.0769 & 0.0021 & \\
\hline
\end{tabular}

Within the same row, groups with the same lowercase were not statistically significant. Within the same column, groups with the same uppercase were not statistically significant

Table 1: Volume of remaining filling material (mean $\pm \mathrm{SD}$ ) after basic and supplementary retreatment techniques in all groups along the entire root canal length

\begin{tabular}{|c|c|c|c|c|c|c|c|c|}
\hline & \multicolumn{3}{|c|}{ Basic retreatment technique (a) } & \multirow[b]{2}{*}{$p$ value } & \multicolumn{3}{|c|}{ Supplementary retreatment technique (b) } & \multirow[b]{2}{*}{$p$ value } \\
\hline & Group I & Group II & Group III & & Group I & Group II & Group III & \\
\hline Apical & $8.28 \pm 1.45^{\mathrm{A}, \mathrm{a}}$ & $8.26 \pm 2.57^{C, a}$ & $8.49 \pm 1.63^{1, a}$ & 0.9788 & $5.48 \pm 0.88^{\mathrm{E}, \mathrm{b}}$ & $4.65 \pm 1.06^{\mathrm{G}, \mathrm{b}}$ & $1.55 \pm 0.47^{\mathrm{K}, \mathrm{c}}$ & $<0.0001$ \\
\hline Middle & $10.30 \pm 0.94^{\mathrm{A}, \mathrm{d}}$ & $10.58 \pm 1.35^{\mathrm{C}, \mathrm{d}}$ & $10.69 \pm 1.02^{1, d}$ & 0.8547 & $6.44 \pm 1.54^{\mathrm{E}, \mathrm{F}, \mathrm{e}}$ & $5.54 \pm 1.08^{\mathrm{G}, \mathrm{e}}$ & $3.48 \pm 0.90^{L, f}$ & 0.0064 \\
\hline Coronal & $14.43 \pm 1.49^{\mathrm{B}, \mathrm{g}}$ & $14.72 \pm 1.51^{\mathrm{D}, \mathrm{g}}$ & $14.64 \pm 1.71^{J, g}$ & 0.9550 & $8.17 \pm 0.89^{\mathrm{F}, \mathrm{h}}$ & $7.56 \pm 1.12^{\mathrm{H}, \mathrm{h}}$ & $4.32 \pm 0.96^{L, j}$ & 0.0001 \\
\hline$p$ value & $<0.0001$ & 0.0005 & $<0.0001$ & & 0.0094 & 0.0034 & 0.0005 & \\
\hline
\end{tabular}

Within the same row, groups with the same lowercase were not statistically significant. Within the same column, groups with the same uppercase were not statistically significant 
Table 3: Mean and standard deviation of \% reduction of root filling in all groups along the entire root canal length

\begin{tabular}{lcccr}
\hline & \multicolumn{3}{c}{ \% Reduction of root fillings } & \\
\cline { 2 - 4 } & Group I & Group II & Group III & p value \\
\hline Apical & $31.63 \pm 17.94^{\mathrm{F}, \mathrm{c}}$ & $38.22 \pm 26.14^{\mathrm{G}, \mathrm{c}}$ & $81.19 \pm 6.42^{\mathrm{H}, \mathrm{d}}$ & 0.0024 \\
Middle & $37.41 \pm 14.89^{\mathrm{F}, \mathrm{g}}$ & $45.78 \pm 16.15^{\mathrm{G}, \mathrm{g}, \mathrm{h}}$ & $67.75 \pm 5.52^{\mathrm{l}, \mathrm{h}}$ & 0.0089 \\
Coronal & $43.13 \pm 6.09^{\mathrm{F}, \mathrm{k}}$ & $48.46 \pm 5.57^{\mathrm{G}, \mathrm{k}}$ & $70.45 \pm 5.42^{\mathrm{l}, \mathrm{m}}$ & $<0.0001$ \\
$p$ value & 0.4500 & 0.6575 & 0.0077 & \\
\hline
\end{tabular}

Within the same row, groups with the same lowercase were not statistically significant. Within the same column, groups with the same uppercase were not statistically significant

The study found that the existing root canal filling could not be entirely removed after basic treatment due to Endosequence BC Sealer adhesion to root canal dentine and the unique morphology of oval-shaped canals, which makes existing filling removal difficult, in agreement with the results of Hammad et al., ${ }^{9}$ Roggendorf et al., ${ }^{10}$ Abramovitz et al., ${ }^{11}$ Ma et al., ${ }^{12}$ Rödig et al., ${ }^{13}$ and Kaşıkçı Bilgi et al. ${ }^{8}$ The percentages of remaining filling material were substantially higher for the basic PTUR retreatment because the apical diameter of the prepared root canal $(\# 30,0.09)$ is larger than the tip of the D3 file $(\# 20,0.07)$. The instruments work close to the filling material and the dentin walls, just as they do in retreatment conditions.

Following the use of XPF-R files, TRN, and WOG file systems, the volume of residual filling material was minimized by $73.13,44.15$, and $37.39 \%$, respectively $(p<0.05)$. Therefore, the hypothesis was rejected since there was a significant difference in the outcomes of each file system used.

The results showed significantly less residual filling material when XPF-R was utilized following the initial retreatment procedure, which indicated an efficient way to eliminate the residual filling material. These results are attributed to the instrument adaptive core technology, tremendous flexibility, and snakelike design, which allows it to change the shape as it expands and contracts during the rotation within the canal, reaching unreachable parts of the canal and eventually cleaning the canal surface, ${ }^{19}$ which is in harmony with the results of Silva et al., ${ }^{6}$ Aksel et al., ${ }^{20}$ Kapasi et al., ${ }^{15}$ and Tavares et al. ${ }^{21}$ Moreover, XPF-R instrument is made of an innovative alloy (MaxWire; FKG) that permits it to expand at body temperature. Hence, the elliptical part of the instrument is compressed by the resistance imposed by canal anatomy, forcing the tip of the file against the canal walls during use. So, the instrument's semi-active tip can abrade the dentinal walls after it expands inside the canal area, and hence, it can remove root filling materials even in an unreachable area. ${ }^{22}$ Although the complicated structure (lateral canals, apical ramifications, and irregular apical foramen) of the apical third is the most crucial area for thorough cleaning in the root canal. ${ }^{17}$ It was observed that in the coronal and middle thirds, the volume of remaining filling material was significantly higher than in the apical third. This finding was due to the larger tip diameter (\#30) of XPF-R that cleans better in the apical region.

TRN effectiveness in eliminating root filling residues from ovalshaped canals has not been tested. As a result, no direct comparison is feasible. On the contrary, the current results revealed that using this supplementary single file effectively removed the residual filling material by $44.15 \%$. The results of TRN can be due to their slim shape, which allows for more excellent debridement space. In addition to that, the cross-section of TRN instrument is designed with an off-center parallelogram. Because of the instrument geometry, regressive tapers, slenderized pattern, and the heat-treatment of the NiTi alloy, it has been stated that TRN files preserve the structural dentin and the tooth entirety and allow the removal of filling residue until the coronal third. ${ }^{23,25}$ TRN had a slightly higher cleaning efficiency than WOG; nonetheless, the difference was not significant. This finding could be due to the WOG larger taper (size 25, taper 0.07) than due to TRN (size 36, 0.03 taper).

WOG files are featured by a parallelogram-shaped crosssection, which provides an efficient space for better coronal debris cutting and transportation. ${ }^{15}$ However, the current results revealed an increased percentage of root canal filling remaining within the root canal space with WOG. Consequently, it was shown to be the least effective supplementary option for endodontic retreatment in this investigation. These results are in harmony with the finding of Bago et al. ${ }^{17}$ and Azim et al. ${ }^{18}$

The lower cleaning efficiency of the WOG file could be attributed to several factors, including its design and motion kinematics. The continuous motion of the rotary files favors upward debris removal along with the flutes. In contrast, the backward motion of the reciprocating files causes debris to be compacted along the dentinal walls and pressed into the lateral canals. ${ }^{28}$ It also features three cutting edges with radial lands to support the blades and small chip space. The smaller the instrument chip space, the less effective is the removal of debris. ${ }^{29}$ Furthermore, the presence of radial lands tends to burnish the cut dentin on the root canal wall; active cutting edge instruments have a higher debris removal capability than instruments with radial lands. ${ }^{30}$

In the current study, the use of supplementary single-file systems enhanced the removal of residual root canal filling materials after the basic retreatment with PTUR retreatment system in the oval-shaped canal in the following descending order: XPF-R, TRN, and then WOG. However, the findings may not be applicable in all situations since only one obturation technique was used and oval-shaped root canals were only chosen as samples.

\section{Conclusion}

With regard to the scope of the limitations of this study, it was determined that none of the commercially marketed supplementary retreatment approaches can eliminate the remaining filling material totally from the root canal. However, using the XPF-R instrument as a supplementary substantially enhanced the removal of existing filling material followed by the TRN system. 


\section{References}

1. Nair P. On the causes of persistent apical periodontitis: a review. Int Endod J 2006;39(4):249-281. DOI: 10.1111/j.1365-2591.2006.01099.x. PMID: 16584489.

2. Hülsmann M, Drebenstedt $S$, Holscher C. Shaping and filling root canals during root canal re-treatment. Endod Topics 2011;19(1): 74-124. DOI: 10.1111/j.1601-1546.2011.00264.x.

3. Cavenago B, Ordinola-Zapata R, Duarte M, et al. Efficacy of xylene and passive ultrasonic irrigation on remaining root filling material during retreatment of anatomically complex teeth. Int Endod J 2014;47(11):1078-1083. DOI: 10.1111/iej.12253.

4. Gorni F, Gagliani M. The outcome of endodontic retreatment: a 2-yr follow-up. J Endod J 2004;30(1):1-4. DOI: 10.1097/00004770200401000-00001.

5. Silva E, Belladonna F, Carapiá M, et al. Micro-computed tomographic evaluation of canal retreatments performed by undergraduate students using different techniques. Restor Dent Endod 2018;43(1):e5. DOI: 10.5395/rde.2018.43.e5.

6. Silva E, Belladonna F, Zuolo A, et al. Effectiveness of XP-endo Finisher and $X P$-endo Finisher $R$ in removing root filling remnants: a micro-CT study. Int Endod J 2018;51(1):86-91. DOI: 10.1111/iej.12788.

7. de Siqueira Zuolo A, Zuolo M, da Silveira Bueno C, et al. Evaluation of the efficacy of TRUShape and Reciproc file systems in the removal of root filling material: an ex vivo micro-computed tomographic study. J Endod 2016;42(2):315-319. DOI: 10.1016/j.joen.2015.11.005

8. Kaşıkçı Bilgi I, Köseler I, Güneri P, et al. Efficiency and apical extrusion of debris: a comparative ex vivo study of four retreatment techniques in severely curved root canals. Int Endod J 2017;50(9):910-918. DOI: 10.1111/iej.12708.

9. Hammad M, Qualtrough A, Silikas N. Three-dimensional evaluation of effectiveness of hand and rotary instrumentation for retreatment of canals filled with different materials. J Endod 2008;34(11):1370-1373. DOI: 10.1016/j.joen.2008.07.024.

10. Roggendorf M, Legner M, Ebert J, et al. Micro-CT evaluation of residual material in canals filled with Activ GP or GuttaFlow following removal with NiTi instruments. Int Endod J 2010;43:200-209. DOI: 10.1111/j.1365-2591.2009.01659.x.

11. Abramovitz I, Relles-Bonar S, Baransi B, et al. The effectiveness of a self-adjusting file to remove residual gutta-percha after retreatment with rotary files. Int Endod J 2012;45(4):386-392. DOI: 10.1111/j.13652591.2011.01988.x.

12. Ma J, Al-Ashaw A, Shen $Y$, et al. Efficacy of ProTaper Universal Rotary Retreatment system for gutta-percha removal from oval root canals: a micro-computed tomography study. J Endod 2012;38(11):1516-1520. DOI: 10.1016/j.joen.2012.08.001.

13. Rödig T, Hausdörfer T, Konietschke F, et al. Efficacy of D-RaCe and ProTaper Universal Retreatment NiTi instruments and hand files in removing gutta-percha from curved root canals-a microcomputed tomography study. Int Endod J 2012;45(6):580-589. DOI: 10.1111/j.1365-2591.2012.02014.x.

14. Özyürek T. Cyclic Fatigue Resistance of Reciproc, WaveOne, and WaveOne Gold Nickel-Titanium Instruments. J Endod 2016;42(10):1536-1539. DOI: 10.1016/j.joen.2016.06.019.

15. Kapasi $K$, Kesharani $P$, Kansara $P$, et al. In vitro comparative evaluation of efficiency of XP-endo shaper, XP-endo finisher, and XP-endo finisher-R files in terms of residual root filling material, preservation of root dentin, and time during retreatment procedures in oval canals-a cone-beam computed tomography analysis. J Conserv Dent 2020;23(2):145-151. DOI: 10.4103/JCD.JCD_257_20.
16. Gavini G, Caldeira CL, Akisue E, et al. Resistance to flexural fatigue of Reciproc R25 files under continuous rotation and reciprocating movement. J Endod 2012;38(5):684-687. DOI: 10.1016/ j.joen.2011.12.033.

17. Bago I, Plotino $G$, Katic M, et al. Evaluation of filling material remnants after basic preparation, apical enlargement, and final irrigation in retreatment of severely curved root canals in extracted teeth. Int Endod J 2020;53(7):962-973. DOI: 10.1111/iej.13287.

18. Azim A, Wang H, Tarrosh M, et al. Comparison between single-file rotary systems: Part 1-efficiency, effectiveness, and adverse effects in endodontic retreatment. J Endod 2018;44(11):1720-1724. DOI: 10.1016/j.joen.2018.07.022.

19. Machado A, Guilherme B, Provenzano J, et al. Effects of preparation with the Self-Adjusting File, TRU Shape and XP-endo Shaper systems, and a supplementary step with XP-endo Finisher R on filling material removal during retreatment of mandibular molar canals. Int Endod J 2019;52(5):709-715. DOI: 10.1111/iej.13039.

20. Aksel H, Küçükkaya Eren S, Askerbeyli Örs S, et al. Micro-CT evaluation of the removal of root fillings using the ProTaper Universal Retreatment system supplemented by the XP-Endo Finisher file. Int Endod J 2019;52(7):1070-1076. DOI: 10.1111/iej.13094.

21. Tavares S, Gomes C, Marceliano-Alves M, et al. Supplementing filling material removal with XP-Endo Finisher R or R1-Clearsonic ultrasonic insert during retreatment of oval canals from contralateral teeth. Aust Endod J 2021;47(2):188-194. DOI: 10.1111/aej.12451.

22. De-Deus G, Belladonna F, Zuolo A, et al. XP-endo Finisher R instrument optimizes the removal of root filling remnants in oval-shaped canals. Int Endod J 2019;52(6):899-907. DOI: 10.1016/ j.ortho.2021.01.009.

23. Van der Vyver $\mathrm{P}$, Vorster $\mathrm{M}$, Peters $\mathrm{O}$. Minimally invasive endodontics using a new single-file rotary system. Int Dent-African Ed 2019;9(4): 6-20. Available from: https://www.moderndentistrymedia.com/ moderndentistrymedia/wp-content/uploads/2020/04/van-dervyver.pdf.

24. Dentsply Sirona. TruNatomy Brochure [Internet]. Johnson (TN): Dentsply Sirona; 2010. Available from: https://www.dentsplysirona. com/en/explore/endodontics/trunatomy.html.

25. Riyahi M, Bashiri A, Alshahrani K, et al. Cyclic fatigue comparison of TruNatomy, Twisted File, and ProTaper Next Rotary Systems. Int J Dent 2020:3190938. DOI: 10.1155/2020/3190938.

26. De-Deus G, Marins J, Silva E, et al. Accumulated hard tissue debris produced during reciprocating and rotary nickel-titanium canal preparation. J Endod 2015;41(5):676-681. DOI: 10.1016/ j.joen.2014.11.028.

27. Wu M, Wesselink P. A primary observation on the preparation and obturation of oval canals. Int Endod J 2001;34(2):137-141. DOI: 10.1046/j.1365-2591.2001.00361.x.

28. Robinson J, Lumley $\mathrm{P}$, Cooper $\mathrm{P}$, et al. Reciprocating root canal technique induces greater debris accumulation than a continuous rotary technique as assessed by 3 -dimensional microcomputed tomography. J Endod 2013;39(8):1067-1070. DOI: 10.1016/ j.joen.2013.04.003.

29. Bürklein S, Hinschitza K, Dammaschke T, et al. Shaping ability and cleaning effectiveness of two single-file systems in severely curved root canals of extracted teeth: Reciproc and WaveOne versus Mtwo and ProTaper. Int Endod J 2012;45(5):449-461. DOI: 10.1111/j.13652591.2011.01996.x

30. Hülsmann M, Peters $\mathrm{O}$, Dummer P. Mechanical preparation of root canals: shaping goals, techniques and means. Endod Topics 2005;10(1):30-76. DOI: 10.1111/j.1601-1546.2005.00152.x. 